\title{
Percutaneous transhepatic biliary drainage complicated with hepatic hydrothorax
}

\author{
Pei-Ming Huang, MD, MS, Taipei, Taiwan
}

Percutaneous transhepatic biliary drainage (PTBD) is a well-established method in the treatment of malignant obstructive jaundice (used in almost $70 \%$ of cases) ${ }^{1,2}$ when endoscopic alternatives fail. However, little attention has been focused on the catheter-related thoracic complications associated with this procedure. Here, we present the first case of a patient who developed hepatic hydrothorax after undergoing PTBD.

\section{CLINICAL SUMMARY}

A 41-year-old man with a history of obstructive jaundice and liver metastasis from undifferentiated nasopharyngeal cancer presented to an institution in Taipei, Taiwan. On abdominal sonography examination, the biliary tract was found to be slightly dilated because of a 2-cm lymphadenopathy at the middle of the common bile duct. He was initially referred for endoscopic drainage; however, there was no change in his condition after this procedure. Therefore, PTBD was performed to address his obstructive jaundice. An 11-hole pigtail catheter

From the Division of Thoracic Surgery, Department of Surgery, National Taiwan University Hospital, and National Taiwan University College of Medicine, Taipei, Taiwan.

Disclosures: Author has nothing to disclose with regard to commercial support.

Received for publication July 28, 2012; revisions received Aug 22, 2012; accepted for publication Dec 11, 2012; available ahead of print Jan 14, 2013.

Address for reprints: Pei-Ming Huang, MD, MS, Division of Thoracic Surgery, Department of Surgery, National Taiwan University Hospital, 7, Chung-Shan S Rd, Taipei 10002, Taiwan (E-mail: e370089@gmail.com).

J Thorac Cardiovasc Surg 2013;145:e34-5

$0022-5223 / \$ 36.00$

Copyright $(c) 2013$ by The American Association for Thoracic Surgery

http://dx.doi.org/10.1016/j.jtcvs.2012.12.039 and a 32-hole ring catheter were used, respectively, for intrahepatic and extrahepatic drainage because of their low dislocation rates.

However, dyspnea was noted 3 weeks later. The patient was unable to lie flat. Computed tomography showed PTBD through the right pleural cavity, bilateral pleural effusion, and ascites (Figure 1). Thoracentesis was performed 5 times and paracentesis was performed once. The PTBD catheter was removed and the tract was packed with 0.035 -in Tornado embolization coils (4 coils $3 \times 40$ $\mathrm{mm}$; Cook Corporation, Bloomington, Ind) then embolized with Gelfoam (Pfizer Corporation, New York, NY). However, a series of sonographic and plain chest radiographic images showed persistent massive pleural effusion on the right side. Meanwhile, we also observed a dilated intrahepatic duct and elevated bilirubin level. Biliary tract stenting via endoscopic retrograde cholangiopancreatography was then performed. Because of the persistent pleural effusion and impending respiratory failure, we performed thoracoscopic repair of diaphragmatic defects. Under general anesthesia, the thoracoscopic instruments were inserted through 3 trocars: $21.5-\mathrm{cm}$ trocars (1 anterior to the fifth intercostal space and the other posterior to the fourth intercostal space) and a 10-mm trocar (at the ninth intercostal space in the midaxillary line) for insertion of the thoracoscope. We detected 1 tract lesion with partial tract wall defect between the diaphragm and the chest wall (Figure 2, $A)$. Furthermore, endoscopic clips were used for obstruction of this tract (Figure 2,B). Chest radiography showed right lung expansion and mild infiltration on postoperative day 1; from this observation, we diagnosed re-expansion edema. The patient's general condition was stable, and
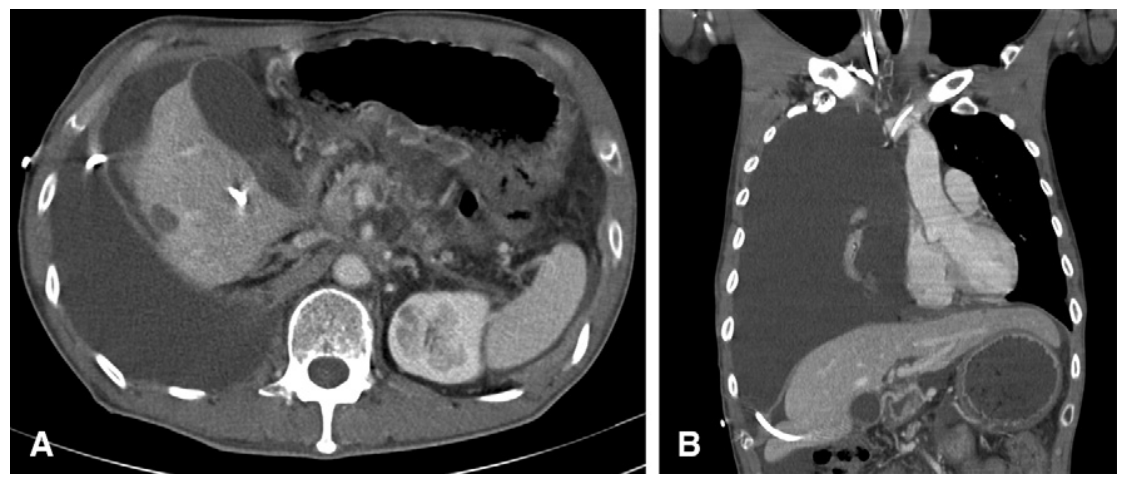

FIGURE 1. A, Chest computed tomography image showing right pleural effusion with ascites, surrounding a linear hyperdensity representing the catheter tip. B, The intrathoracic segment of the catheter entering the diaphragm and hepatic parenchyma. 

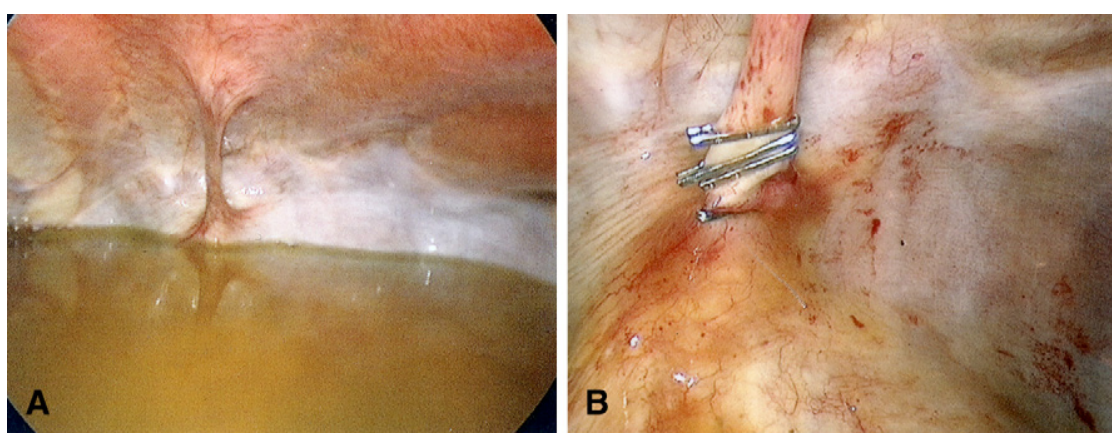

FIGURE 2. A, Thoracoscopy shows 1 tract lesion and partial tract wall defect between the tendinous area of the diaphragm and the chest wall. B, Endoscopic clipping for obstruction of this tract.

he received further chemotherapy to prevent malignant recurrence.

\section{DISCUSSION}

PTBD was introduced $>60$ years ago, and it was performed for postoperative decompression $(59 \%$ of cases reported) and for palliative decompression of obstruction (35\% of cases reported), particularly in cases of malignant disease. ${ }^{2}$ Retrospective evaluation of patients who underwent this procedure showed good results with a low rate of serious complications, such as sepsis $(1.9 \%)$, peritonitis $(0.5 \%)$, severe bleeding $(0.5 \%)$, and procedure-related death $(0.8 \%)$. These results are comparable to those obtained for the endoscopic approach, which has a complication rate of $3.6 \%$ to $14 \%$ and a mortality rate of $0.5 \% .^{1-3}$ Catheter dislocation, which accounted for $8.5 \%$ of the reported cases, was prevented by using self-retaining catheters. ${ }^{3}$ Hepatic hydrothorax is a rare complication in patients undergoing PTBD and is usually associated with high mortality. Operative closure of diaphragmatic defects should be attempted in each patient because spontaneous closure is rare. The fenestrations in the diaphragm allow peritoneopleural communication. ${ }^{4}$ Video-assisted thoracoscopic surgery allows excellent localization of diaphragmatic defects. ${ }^{4}$ Our study provides images of a gross appearance of diaphragmatic tubular defects in patients experiencing hepatic hydrothorax after PTBD. In view of this, additional early thoracoscopic surgery should be beneficial for these patients if other conventional techniques, including pleurodesis or tract embolization, failed. ${ }^{4}$ In patients with ascites, a diaphragmatic injury should be suspected when massive pleural effusion is noted after PTBD; pleurodesis is not preferred in such cases because a large amount of fluid may gush out through the tract. ${ }^{5}$ Apart from surgery, the option is tract embolization. Generally, the technique of transcatheter embolization after PTBD was used for hepatic arterial injury with success rates of $95.8 \%$. However, no literature reports the option of tract embolization for PTBD complicated with hepatic hydrothorax. Besides, the effectiveness of tract embolization may be also limited in cases of patients with massive ascites.

\section{CONCLUSIONS}

Although diaphragm injury is rare, it should always be considered in patients with pleural effusion who have undergone PTBD. Repair of diaphragmatic defects by videoassisted thoracoscopic surgery can help achieve long-term cure.

\section{References}

1. Oberholzer K, Pitton MB, Mildenberger P, Lechner C, Duber C, Thelen M. The current value of percutaneous transhepatic biliary drainage. Rofo. 2002;174: 1081-8.

2. Gunther RW, Schild H, Thelen M. Percutaneous transhepatic biliary drainage: experience with 311 procedures. Cardiovasc Intervent Radiol. 1988;11:65-71.

3. Joseph PK, Bizer LS, Sprayregen SS, Gliedman ML. Percutaneous transhepatic biliary drainage. Results and complications in 81 patients. JAMA. 1986;255: 2763-7.

4. Huang PM, Chang YL, Yang CY, Lee YC. The morphology of diaphragmatic defects in hepatic hydrothorax: thoracoscopic finding. I Thorac Cardiovasc Surg. 2005;130:141-5.

5. Liu WL, Kuo PH, Ku SC, Huang PM, Yang PC. Impact of therapeutic interventions on survival of patients with hepatic hydrothorax. J Formos Med Assoc. 2010;109:582-8. 